PRA-HEP-92/18

\title{
Quantum braided groups
}

\author{
Ladislav HLavatÝ \\ Institute of Physics, Czechoslovak Academy of Sciences \\ and \\ Department of Physics, \\ Faculty of Nuclear Sciences and Physical Engineering \\ Prague, Czechoslovakia
}

\begin{abstract}
A new type of algebras that represent a generalization of both quantum groups and braided groups is defined. These algebras are given by a pair of solutions of the Yang-Baxter equation that satisfy some additional conditions. Several examples are presented.
\end{abstract}

\footnotetext{
${ }^{1}$ Postal address: Břehová 7, 110 Prague 1, Czechoslovakia. E-mail: HLAVATY@FJFI.CVUT.CS
} 


\section{Introduction - quantum groups and braided groups}

Matrix groups like $G L(n), S O(n)$ e.t.c. were generalized in two ways recently. Both are based on deformation of the algebra of functions on the groups generated by coordinate functions $T_{i}^{j}$ that commute

$$
T_{i}^{j} T_{k}^{l}=T_{k}^{l} T_{i}^{j} \quad \Leftrightarrow \quad T_{1} T_{2}=T_{2} T_{1}
$$

In the quantum groups [1, 2] these commutation relations are modified by a matrix $R=\left\{R_{i j}^{k l}\right\}$ so that the functions do not commute but satisfy the relations

$$
R_{12} T_{1} T_{2}=T_{2} T_{1} R_{12}
$$

In this relation the elements of matrix $R$ are numbers but the matrix $T=\left\{T_{i}^{j}\right\}$ is formed by generally noncommuting elements of an algebra.

Another type of deformation of the relations (1) represent the so called braided groups [3] defined by the relation

$$
T_{1} Z_{12} T_{2} Z_{12}^{-1}=Z_{21}^{-1} T_{2} Z_{21} T_{1}
$$

where $Z$ is again a matrix $\left\{Z_{i j}^{k l}\right\}$ with number elements.

The quantum groups appeared to be hidden symmetries of many physical models. The relevance of the braided groups for the low-dimensional quantum field theory was explained in [4].

The goal of this paper is to define a concept that unifies both the quantum groups and braided groups. We call these more general objects quantum braided groups. Before doing that let us summarize the properties of quantum groups and braided groups.

Both the algebras defined by (2) or (3) can be extended to bialgebras with matrix coproduct and counit

$$
\Delta\left(T_{i}^{j}\right):=T_{i}^{k} \otimes T_{k}^{j}, \epsilon\left(T_{i}^{j}\right):=\delta_{i}^{j} .
$$

However, the tensor products of the algebras defined by the relations (2) differ from those defined by the relations (3). The multiplication in the tensor product $A \otimes A$ of the algebras $A$ defined by the relations (2) (corresponding to quantum groups) is

$$
\begin{aligned}
& m_{(A \otimes A)}: A \otimes A \otimes A \otimes A \rightarrow A \otimes A \\
& m_{(A \otimes A)}=(m \otimes m) \circ\left(i d \otimes \tau_{23} \otimes i d\right)
\end{aligned}
$$

where $m$ is the product in $A$ and $\tau_{23}$ is the transposition of the second and third factor in $A \otimes A \otimes A \otimes A$. It is then easy to prove that $A$ is a bialgebra.

On the other hand the multiplication in the tensor product $B \otimes B$ of the algebras $B$ defined by the relations (3) (corresponding to braided groups) is more 
complicated because instead of the simple transposition $\tau$ a more general map $\psi: B \otimes B \rightarrow B \otimes B$ called braiding appears in the product [3].

$$
\begin{gathered}
m_{(B \otimes B)}: B \otimes B \otimes B \otimes B \rightarrow B \otimes B \\
m_{(B \otimes B)}:=(m \otimes m) \circ\left(i d \otimes \psi_{23} \otimes i d\right)
\end{gathered}
$$

where $m$ is the product in $B$ and

$$
\psi\left(T_{i}^{m} \otimes T_{k}^{n}\right):=\psi_{i k}^{m n}{ }_{\text {,rs }}^{j l}\left(T_{j}^{r} \otimes T_{l}^{s}\right)
$$

where

$$
\psi_{i k}^{m n}{ }_{i r s}^{j l}:=Z_{i d}^{a j}\left(Z^{-1}\right)_{a r}^{l b} Z_{s b}^{c n} \tilde{Z}_{c k}^{m d}
$$

and $\tilde{Z}:=\left(\left(Z^{t_{2}}\right)^{-1}\right)^{t_{2}}$.

To prove that $B$ is bialgebra namely that $\Delta$ and $\epsilon$ are morphisms of the algebra $B$ and $B \otimes B$ is a bit more complicated than for the quantum groups but there are no principal problems. The identities

$$
\tilde{Z}_{i l}^{k n} Z_{k j}^{m l}=Z_{i l}^{k n} \tilde{Z}_{k j}^{m l}=\delta_{i}^{m} \delta_{j}^{n}
$$

which follow immediately from the definition of $\tilde{Z}$, is used for that. If antipodes on the bialgebras are defined we get Hopf algebras.

\section{Quantum braided groups}

As mentioned in the beginning, our goal is to define an object that will unify the properties of both quantum and braided groups or more precisely, that will contain both of them as special cases. Prototypes for that are quantum supergroups.

The supergroups are special cases of the braided groups where $Z=\eta:=$ $\operatorname{diag}(+,+, \ldots,-,-, \ldots)$ and $\psi(x \otimes y)=(-)^{|x||y|} y \otimes x$. The defining relations of a quantum supergroup can be written in a form that reminds (2) but the supercommuting nature of its elements is expressed by inclusion of the matrix $\eta$ into the defining relation [5]

$$
\mathcal{R}_{12} T_{1} \eta_{12} T_{2} \eta_{12}=\eta_{12} T_{2} \eta_{12} T_{1} \mathcal{R}_{12}
$$

Comparing (2), (3) and (10) leads us quite naturally to the investigation of algebras given by a pair of $n^{2} \times n^{2}$ matrices $(\mathcal{R}, Z)$ that define relations

$$
\mathcal{R}_{12} T_{1} Z_{12} T_{2} Z_{12}^{-1}=Z_{21}^{-1} T_{2} Z_{21} T_{1} \mathcal{R}_{12}
$$

which include the cases of both quantum and braided groups.

The experience with the quantum groups, braided groups, and quantum supergroups teaches us that the matrices $\mathcal{R}$ and $Z$ cannot be arbitrary but will be restricted by conditions of Yang-Baxter type. These conditions follow from two 
possible ways to transpose a triple of generators $T_{i}^{j}$. In order that the relations (11) can be applied we shall consider triples of the form

$$
T_{1} Z_{12} T_{2} Z_{12}^{-1} Z_{23} Z_{13} T_{3} Z_{13}^{-1} Z_{23}^{-1}
$$

It can be transposed to an expression with the transposed order of $T_{1}, T_{2}, T_{3}$ if matrices $\mathcal{R}$ and $Z$ are invertible and satisfy

$$
\begin{aligned}
Z_{12} Z_{13} Z_{23} & =Z_{23} Z_{13} Z_{12}, \\
\mathcal{R}_{12} Z_{23} Z_{13} & =Z_{23} Z_{13} \mathcal{R}_{12}, \\
Z_{12} Z_{13} \mathcal{R}_{23}^{-1} Z_{32}^{-1} & =\mathcal{R}_{23}^{-1} Z_{32}^{-1} Z_{13} Z_{12}
\end{aligned}
$$

Under these conditions the expression (12) can be transposed by two ways and we require that the results be equal

$$
\begin{gathered}
\mathcal{R}_{12}^{-1} Z_{21}^{-1} \mathcal{R}_{13}^{-1} Z_{31}^{-1} \mathcal{R}_{23}^{-1} Z_{32}^{-1} T_{3} Z_{32} T_{2} Z_{31} Z_{21} T_{1} \mathcal{R}_{23} Z_{23} \mathcal{R}_{13} Z_{13} \mathcal{R}_{12} Z_{13}^{-1} Z_{23}^{-1} \\
\mathcal{R}_{23}^{-1} Z_{32}^{-1} \mathcal{R}_{13}^{-1} Z_{31}^{-1} \mathcal{R}_{12}^{-1} Z_{21}^{-1} T_{3} Z_{32} T_{2} Z_{31} Z_{21} T_{1} \mathcal{R}_{12} Z_{12} \mathcal{R}_{13} Z_{13} \mathcal{R}_{23} Z_{13}^{-1} Z_{12}^{-1}
\end{gathered}
$$

In order that the equation (16) does not impose additional relations for $T$ we require that the matrix $\mathcal{R}$ satisfy the "braided Yang-Baxter equations"

$$
\begin{aligned}
\mathcal{R}_{12} Z_{12} \mathcal{R}_{13} Z_{13} \mathcal{R}_{23} Z_{23} & =\mathcal{R}_{23} Z_{23} \mathcal{R}_{13} Z_{13} \mathcal{R}_{12} Z_{12} \\
\mathcal{R}_{12}^{-1} Z_{21}^{-1} \mathcal{R}_{13}^{-1} Z_{31}^{-1} \mathcal{R}_{23}^{-1} Z_{32}^{-1} & =\mathcal{R}_{23}^{-1} Z_{32}^{-1} \mathcal{R}_{13}^{-1} Z_{31}^{-1} \mathcal{R}_{12}^{-1} Z_{21}^{-1}
\end{aligned}
$$

Introducing $R:=\mathcal{R} Z$ we immediately see that (17) is the ordinary YangBaxter equation (YBE) for $R$

$$
R_{12} R_{13} R_{23}=R_{23} R_{13} R_{12}
$$

and the equations (14) and (15) can be rewritten to simpler forms

$$
\begin{aligned}
& R_{12} Z_{13} Z_{23}=Z_{23} Z_{13} R_{12} \\
& Z_{12} Z_{13} R_{23}=R_{23} Z_{13} Z_{12}
\end{aligned}
$$

The equation (18) is then satisfied due to

Lemma: If $R$ and $Z$ are solutions of the YBE that satisfy (20) and (21) then $P Z P R Z^{-1}$ and $Z^{-1} R P Z P$, where $\mathrm{P}$ is the permutation matrix $P_{i j}^{k l}=\delta_{i}^{l} \delta_{j}^{k}$, are also solutions of the YBE.

Proof can be done by direct check. Let us note that the condition $P Z P Z^{-1}=$ 1 required in [6] is not necessary here.

Conclusion then is that when we have a pair $(R, Z)$ of solutions of the YBE that satisfy the relations $(20)$, (21), we can define the algebra

$$
B(R, Z):=C<T_{i}^{j}>_{i, j=1}^{n} /\left\{R_{a b}^{c d} Z_{c d}^{-1}{ }^{g h} T_{g}^{i} Z_{i h}^{m l} T_{l}^{k}-Z_{b a}^{-1}{ }^{j i} T_{j}^{g} Z_{g i}^{h c} T_{c}^{d} R_{d h}^{m k}\right\}_{a, b, m, k=1}^{n}
$$


that do not impose additional relations of cubic or higher degree. The compact form of the relations in (22) is

$$
R_{12} Z_{12}^{-1} T_{1} Z_{12} T_{2}=Z_{21}^{-1} T_{2} Z_{21} T_{1} R_{12}
$$

that is equivalent to (11).

One can show that matrix coproduct and counit (đ) are morphisms of $B(R, Z)$ into $B(R, Z) \otimes B(R, Z)$ where the product in $B(R, Z) \otimes B(R, Z)$ is defined by (6), (8). To do that one must prove that

$$
R_{12} Z_{12}^{-1} \Delta\left(T_{1}\right) Z_{12} \Delta\left(T_{2}\right)=Z_{21}^{-1} \Delta\left(T_{2}\right) Z_{21} \Delta\left(T_{1}\right) R_{12}
$$

which is simple but tedious exercise with indices where the identity (9) is used. It means that the algebra (22) can be extended to the bialgebra with the coproduct (4). That enable to define the dual algebra of regular functionals $L^{ \pm}=\left\{L_{i}^{ \pm}{ }^{j}\right\}$ on $B(R, Z)$.

Let

$$
<L_{1}^{ \pm}, T_{2} T_{3} \ldots T_{n}>:=\mathcal{R}_{12}^{ \pm} \mathcal{R}_{13}^{ \pm} \ldots \mathcal{R}_{1 n}^{ \pm}
$$

where

$$
\mathcal{R}_{12}^{+}:=Z_{12} \mathcal{R}_{12} Z_{21}=Z_{12} R_{21}, \quad \mathcal{R}_{12}^{-}:=\mathcal{R}^{-1}=Z_{12} R_{12}^{-1}
$$

and

$$
<a b, c>:=<a \otimes b, \Delta(c)>\text {. }
$$

The dual algebra is then characterized by the relations

$$
\mathcal{R}_{21} L_{1}^{\epsilon} Z_{21} L_{2}^{\sigma} Z_{21}^{-1}=Z_{12}^{-1} L_{2}^{\sigma} Z_{12} L_{1}^{\epsilon} \mathcal{R}_{21}
$$

or equivalently

$$
R_{21} Z_{21}^{-1} L_{1}^{\epsilon} Z_{21} L_{2}^{\sigma}=Z_{12}^{-1} L_{2}^{\sigma} Z_{12} L_{1}^{\epsilon} R_{21}
$$

\section{Solutions of the Yang-Baxter system}

The problem that we have to solve for determination of a quantum braided group is to find solutions of the system $(13,19,20,21)$.

There are several simple solutions of the system. One of them is $Z=1, R$ any solution of the YBE. This gives the algebras that correspond to the ordinary (unbraided) quantum groups [2]. Other solutions are $Z=R$ or $Z=P R^{-1} P, R$ being any solution of the YBE. They correspond to the (unquantised) braided groups.

There are also trivial solutions in the sense that they give no relations for the algebra (22).

Lemma 1: If $Z$ is a multiple of the permutation matrix P i.e. $Z_{i j}^{k l}=z \delta_{i}^{l} \delta_{j}^{k}$ then the only solution of the system (13, 19, 20,21) is such that $R$ is a multiple of the 
permutation matrix and the relation (23) is identity.

Proof: $P$ solves (13) and from (20) and (21) we get $R_{i j}^{k l} \delta_{n}^{m}=R_{j n}^{m k} \delta_{i}^{l}$, solution of what is a multiple of the permutation matrix $R=r P$. The relations (23) then read

$$
T_{1} P_{12} T_{2} P_{12}=P_{12} T_{2} P_{12} T_{1}
$$

that is identity.

Lemma 2: If $R=r P$ then an arbitrary YBE solution $Z$ solves the system $(13,19,20,21)$ and there are no relations in the algebra (22).

Proof: The matrix $r P$ solves (19) and the equations (21) and (20) for $R=P$ are identities so that any solution of (13) solves the system. The relations (23) read

$$
P_{12} Z_{12}^{-1} T_{1} Z_{12} T_{2}=Z_{21}^{-1} T_{2} Z_{21} T_{1} P_{12}
$$

that is also identity.

Question is whether there are solutions of the system $([13,19,20,21)$ that provide nontrivial examples of quantum braided groups. The answer is positive even though it seems that they are relatively rare. The point is that we know a lot of solutions of the YBE at present but only a few of their pairs satisfy (20), (21).

To present some nontrivial examples we are going to solve the system (13, 19, 20, 21) for $n=2$ i.e. for matrices $R$ and $Z$ of the dimension $4 \times 4$. In this dimension we have at our disposal the complete list of the YBE solutions [7] so that in principle it is easy to check whether pairs of the solutions satisfy (20) and (21).

There are two obstacles when we want to solve the system completely at least in this dimension. First, the solutions of the YBE are rather too many. Even if we restrict ourselves to the invertible ones that form eleven classes [8, 9] they give 121 pairs and it takes a lot of time to check them. Second and more important, even if we do that we can anyway miss some solutions. The reason is that the solutions of the YBE are given up to symmetries of the YBE but the cartesian product of the group of symmetries is not the group of symmetry of the system (13, 19,20,21). Indeed, if $R$ and $Z$ solve the system (13, 19,20,21) matrices

$$
\begin{gathered}
R^{\prime}=(V \otimes V) R(V \otimes V)^{-1}, \quad V \in S L(2, C), \\
S^{\prime}=(W \otimes W) S(W \otimes W)^{-1}, \quad W \in S L(2, C),
\end{gathered}
$$

solve the YBE (13) and (19) but not necessarily the equations (20) and (21).

Therefore, to solve the system completely one can take just one solution from the symmetry class (31) but all solutions from the class (32) (or vice versa). This makes the inspection of solutions of the YBE very complicated. Nevertheless checking many solutions of the YBE we have been able to find several solutions of the system $(13,19,20,21)$ that give examples of the quantum braided groups in two dimensions.

A part of the inspection can be made analytically:

Lemma 3: If $Z$ is a diagonal solution of the YBE, $Z=\operatorname{diag}(x, u, v, y)$, xuvy $\neq 0$, 
then there are three types of solutions of the system (13, 19, 20,21):

1) If $x=u=v=y$ then $\mathrm{R}$ is an arbitrary YBE solution.

2) If $x^{2}=u^{2}=v^{2}=y^{2}$ then $\mathrm{R}$ is an arbitrary YBE solution of the eight-or-lessvertex form

$$
R=\left(\begin{array}{llll}
q & 0 & 0 & a \\
0 & r & b & 0 \\
0 & c & s & 0 \\
d & 0 & 0 & t
\end{array}\right)
$$

3) R is an arbitrary YBE solution of the six-or-less-vertex form i.e. (33) where $a=d=0$.

Proof: Let $Z$ is diagonal i.e. $Z_{i j}^{k l}=z_{i j} d_{i}^{k} d_{j}^{l}$. Then we get from (20)

$$
R_{i j}^{k l} z_{k m} z_{l m}=z_{j m} z_{i m} R_{i j}^{k l} \text { no summation. }
$$

Similarly from (21) we get

$$
z_{m i} z_{m j} R_{i j}^{k l}=R_{i j}^{k l} z_{m k} z_{m l} \text { no summation. }
$$

Therefore, if there are $i, j, k, l, m$ such that

$$
z_{k m} z_{l m} \neq z_{j m} z_{i m} \text { or } z_{m i} z_{m j} \neq z_{m k} z_{m l}
$$

then $R_{i j}^{k l}=0$. Substituting $z_{11}=x, z_{12}=u, z_{21}=v, z_{22}=y$. into the condition (35,36) gives the cases 1) - 3).

The case 1) of the lemma 3 gives ordinary quantum groups. The case 2) implies that only eight-or-less-vertex matrices $R$ can be used for quantization of supergroups (cf. [10]). The case 3) will be discussed in the next Section.

\section{Examples}

The first type of nontrivial examples provides us Lemma 3, case 3). From the list of solutions of the YBE on can see that there are just four six-or-less-vertex invertible solutions of the YBE. The first one is $R=r P$ that due to the Lemma 2 gives trivial "no relation" quantum braided group. The other three are

$$
\begin{gathered}
R_{5}=\left(\begin{array}{cccc}
q & 0 & 0 & 0 \\
0 & 1 & 0 & 0 \\
0 & q-t & q t & 0 \\
0 & 0 & 0 & q
\end{array}\right), R_{6}=\left(\begin{array}{cccc}
q & 0 & 0 & 0 \\
0 & 1 & 0 & 0 \\
0 & q-t & q t & 0 \\
0 & 0 & 0 & -t
\end{array}\right) \\
R_{8}=\left(\begin{array}{cccc}
q & 0 & 0 & 0 \\
0 & r & 0 & 0 \\
0 & 0 & s & 0 \\
0 & 0 & 0 & t
\end{array}\right)
\end{gathered}
$$


(The numbering corresponds to that in classifications given in [8, 9].)

The defining relations of the quantum braided group given by $R=R_{5}, Z=$ $\operatorname{diag}(x, u, v, y)$ are

$$
\begin{aligned}
t A B & =\sigma B A, \\
q C A & =\sigma A C, \\
q D B & =\tau B D, \\
t C D & =\tau D C, \\
\sigma \tau B C & =q t C B, \\
\sigma(A D-D A) & =(q-t) C B,
\end{aligned}
$$

where $\sigma=v / x, \tau=y / u$ and $A, B, C, D$ are generators of the algebra.

$$
T=\left(\begin{array}{cc}
A & B \\
C & D
\end{array}\right) .
$$

This quantum braided group have a structure similar to the well known quantum group $G L_{q, s}(2)$ [11] which is obtained when $y=u v / x$.

On the other hand, the quantum braided group given by $R=R_{6}, Z=$ $\operatorname{diag}(x, u, v, y)$ where $q \neq-t$ reminds the quantum supergroup $G L_{q, t}(1 \mid 1)$ [12]. The defining relations are

$$
\begin{aligned}
B^{2} & =0=C^{2} \\
t A B & =\sigma B A, \\
q C A & =\sigma A C, \\
t D B & =-\tau B D, \\
q C D & =-\tau D C, \\
\sigma \tau B C & =q t C B, \\
\sigma(A D-D A) & =(q-t) C B,
\end{aligned}
$$

There are several cases of quantum braided groups given by $R=R_{8}=$ $\operatorname{diag}(q, r, s, t), Z=R_{8}^{\prime}=\operatorname{diag}(x, u, v, y)$ (cf. [13]).

If $q=t, q^{2}=r s$ we get six relations algebra

$$
\begin{aligned}
& A B=\kappa B A, \\
& C A=\kappa A C, \\
& D B=\rho B D, \\
& C D=\rho D C, \\
& C B=\omega B C, \\
& A D=D A,
\end{aligned}
$$


where $\kappa=v q /(x s), \rho=r y /(u t), \omega=r v y /(s x u)$. When one of the above relations between the parameters $q, r, s, t$ does not hold we get additional relations. Namely if $q \neq t$, then

$$
B^{2}=0=C^{2} .
$$

If $q^{2} \neq r s$ then

$$
A B=B A=A C=C A=0 .
$$

If $t^{2} \neq r s$ then

$$
B D=D C=B D=D B=0 .
$$

In the generic case when $q \neq t, q^{2} \neq r s \neq t^{2}$, the quantum braided group is defined by

$$
\begin{gathered}
A B=B A=A C=C A=B D=D C=B D=D B=B^{2}=C^{2}=0, \\
A D=D A, C B=\omega B C .
\end{gathered}
$$

The braiding relations for all the above given quantum braided groups are given by matrix $Z=R_{8}^{\prime}=\operatorname{diag}(x, u, v, y)$ and read

$$
\begin{aligned}
& \psi(A \otimes X)=X \otimes A, \quad \psi(X \otimes A)=A \otimes X, \quad X \in\{A, B, C, D\}, \\
& \psi(B \otimes B)=\xi B \otimes B, \\
& \psi(B \otimes C)=\xi^{-1} C \otimes B, \\
& \psi(C \otimes B)=\xi^{-1} B \otimes C, \\
& \psi(C \otimes C)=\xi C \otimes C, \\
& \psi(D \otimes X)=X \otimes D, \quad \psi(X \otimes D)=D \otimes X, \quad X \in\{A, B, C, D\}
\end{aligned}
$$

where $\xi=x y /(u v)$. Note that $A, D$ are always bosonic. Only $B$ and $C$ can have anomalous statistics for this $Z$.

Other examples provide the solutions of the system (13, 19,20,21) where

$$
Z=R_{10}^{\prime}=\left(\begin{array}{cccc}
1 & 0 & 0 & 0 \\
x & 1 & 0 & 0 \\
y & 0 & 1 & 0 \\
z & y & x & 1
\end{array}\right)
$$

The braiding $\psi$ given by this matrix is

$$
\begin{aligned}
\psi(C \otimes X) & =X \otimes C, \quad \psi(X \otimes C)=C \otimes X, \quad X \in\{A, B, C, D\} \\
\psi(A \otimes A) & =A \otimes A+\tau C \otimes C \\
\psi(A \otimes B) & =B \otimes A-\tau(A-D) \otimes C, \\
\psi(A \otimes D) & =D \otimes A-\tau C \otimes C \\
\psi(B \otimes A) & =A \otimes B-\tau C \otimes(A-D), \\
\psi(B \otimes B) & =B \otimes B+\tau(A-D) \otimes(A-D)+2 \tau^{2} C \otimes C,
\end{aligned}
$$




$$
\begin{aligned}
\psi(B \otimes D) & =D \otimes B+\tau C \otimes(A-D), \\
\psi(D \otimes A) & =A \otimes D-\tau C \otimes C, \\
\psi(D \otimes B) & =B \otimes D+\tau(A-D) \otimes C, \\
\psi(D \otimes D) & =D \otimes D+\tau C \otimes C,
\end{aligned}
$$

where $\tau=z-x y$. Note that there are again two bosonic elements, namely $C$ and $A+D$. For $z=x y$ the braiding is bosonic even though $Z \neq 1$.

There are two solutions of the system $(13,19,20,21)$ with $Z=R_{10}^{\prime}$. The matrix $R$ then is either

$$
R=R_{10}=\left(\begin{array}{llll}
1 & 0 & 0 & 0 \\
g & 1 & 0 & 0 \\
h & 0 & 1 & 0 \\
f & h & g & 1
\end{array}\right) \quad \text { or } \quad R=R_{11}=\left(\begin{array}{cccc}
1 & 0 & 0 & 0 \\
-g & 1 & 0 & 0 \\
g & 0 & 1 & 0 \\
-g h & h & -h & 1
\end{array}\right)
$$

The quantum braided group given by $Z=R_{10}^{\prime}, R=R_{11}$ is defined by the relations

$$
\begin{aligned}
B A & =A B+\chi B^{2} \\
D B & =B D+\gamma B^{2} \\
C B & =B C+\gamma A B-\chi B D \\
C A & =A C+\tau A B+\gamma A(A-D)+\gamma B C+\tau(\chi-\gamma) B^{2}-\gamma \chi B D \\
D C & =C D-\chi(A-D) D+\chi B C-\tau(\chi-\gamma) B^{2}+\left(\tau-\chi^{2}\right) B D \\
D A & =A D+\gamma A B+\chi B D+(\tau+\gamma \chi) B^{2}
\end{aligned}
$$

where $\tau=z-x y, \quad \chi=y-h, \gamma=y-g$. Note that for $\tau=0$ we get the nonstandard unbraided deformation of $G L(2)$ [14, 15] even if $Z \neq 1$.

The last example of the quantum braided group in this paper is given by $R=R_{10}, Z=R_{10}^{\prime}$. We can assume that the parameters of $R_{10}$ satisfy $g+h \neq 0$ or $f \neq g h$ because otherwise we get a special case of the previous example. Under this assumption the defining relations read

$$
\begin{gathered}
A B=B A=D B=B D=B^{2}=0, \\
A D=D A, B C=C B, A^{2}=D^{2}, \\
C A=A C+(y-h) A(A-D)+(y+g) B C \\
C D=D C+(y-h) D(A-D)-(y+g) B C
\end{gathered}
$$

If $g+h \neq 0$ then moreover

$$
B C=A(A-D), \quad A C=C D
$$




\section{Conclusions}

We have written down the defining relations of a new type of algebras that generalize both the quantum groups and braided groups as well as the quantum supergroups. The relations of the algebras are determined by a pair of matrices $(R, Z)$ that solve a system of Yang-Baxter-type equations. The algebras can be extended to bialgebras with matrix coproduct and counit, however, the multiplication in the tensor product of the algebras is defined by virtue of the braiding map given by the matrix $Z$.

Besides simple solutions of the system of the Yang-Baxter-type equations that generate either quantum groups or braided groups, we have found several solutions that generate genuine quantum braided groups in the sense that by a choice of parameters we can get quantum groups or braided groups as a special cases.

This work was supported in part by the grant CSAV No. 11086.

\section{References}

[1] V. G. Drinfeld, Quantum Groups, in Proc. IMS, Berkeley 1986.

[2] N. Yu. Reshetikhin, L. A. Takhtajan, L. D. Faddeev, Algebra i analiz 1 (1989) 178 (in Russian), Leningrad Math. J. 1 (1990) 193.

[3] S. Majid, J. Math. Phys. 32 (1991) 3246.

[4] S. Majid, Lett. Math. Phys. 22 (1991) 167.

[5] Li Liao, X-Ch. Song, Mod. Phys. Lett. 11 (1991) 959.

[6] N. Yu. Reshetikhin, Lett. Math. Phys. 20 (1990) 331.

[7] J. Hietarinta Phys. Lett. A 165 (1992) 245.

[8] L. Hlavatý, J. Phys. A 20 (1987) 1661.

[9] L. Hlavatý, J. Phys. A 25 (1992) L63.

[10] L. Hlavatý, Mod. Phys. Lett. A in press.

[11] Č. Burdík, L. Hlavatý, J. Phys. A 24 (1991) L165.

[12] L. Dabrowski, L. Wang, Phys. Lett B 244 (1990) 227.

[13] L. Hlavatý, J. Phys. A 24 (1991) 2903.

[14] Zakrzewski, Lett. Math. Phys. (1991).

[15] H. Ewen, O. Ogivetski, J. Wess, preprint MPI-PAE/ Pth 18/91. 